\title{
Congestive heart failure associated with POEMS syndrome that was adequately distinguished from cardiac amyloidosis: a case report and literature review
}

\author{
Goshi Hagiwara $^{1} \wedge$, Masahisa Arahata ${ }^{2}$, Kohei Hosokawa ${ }^{1}$, Masaya Shimojima ${ }^{3}$, Shinji Nakao ${ }^{1}$ \\ ${ }^{1}$ Department of Hematology, Kanazawa University Hospital, Kanazawa, Ishikawa, Japan; ${ }^{2}$ Department of Internal Medicine, Nanto Municipal \\ Hospital, Nanto, Japan; ${ }^{3}$ Department of Cardiovascular Medicine, Kanazawa University Graduate School of Medical Sciences, Kanazawa, Japan \\ Correspondence to: Masahisa Arahata. Department of Internal Medicine, Nanto Municipal Hospital, 938 Inami, Nanto, Toyama 932-0211, Japan. \\ Email: rqxhf297@yahoo.co.jp.
}

\begin{abstract}
Congestive heart failure $(\mathrm{CHF})$ is a common complication in patients with AL amyloidosis but is rare in another plasma cell dyscrasia, POEMS syndrome. A 52-year-old man developed POEMS syndrome with a solitary plasmacytoma complicated by CHF mimicking cardiac amyloidosis (CA). His neurological symptoms and CHF did not improve after radiotherapy (50 Gy) targeting the plasmacytoma. Based on typical findings of noninvasive examinations such as elevated serum NT-proBNP $(12,631 \mathrm{pg} / \mathrm{mL})$, a pseudo-infarct pattern on electrocardiography, interventricular septal thickening with a granular sparkling appearance and an apical sparing pattern of longitudinal strain on echocardiography, and late gadolinium enhancement of the left ventricular wall on cardiac magnetic resonance imaging (MRI), severe CA ineligible for autologous peripheral blood stem cell transplantation (auto-PBSCT) was strongly suspected. However, myocardial biopsy failed to reveal amyloid deposits, and CHF markedly improved after only one cycle of chemotherapy with melphalan and dexamethasone. Accordingly, CA was denied as the etiology of his heart failure, and the patient was finally diagnosed with POEMS syndrome. As a result, high-dose melphalan followed by auto-PBSCT improved his neurological symptoms. Careful evaluation is therefore needed to appropriately treat patients with POEMS syndrome complicated by CHF, even when the results of noninvasive examinations are typical for AL amyloidosis.
\end{abstract}

Keywords: POEMS syndrome; congestive heart failure (CHF); amyloid cardiomyopathy; melphalan plus dexamethasone therapy; autologous peripheral blood stem cell transplantation

Submitted Mar 07, 2021. Accepted for publication Jun 20, 2021.

doi: 10.21037/atm-21-1071

View this article at: https://dx.doi.org/10.21037/atm-21-1071

\section{Introduction}

Polyneuropathy, organomegaly, endocrinopathy, monoclonal protein, and skin changes (POEMS) syndrome is characterized by the damage of various organs by plasma cell dyscrasia; however, POEMS syndrome rarely involves the heart. Dispenzieri et al. reported that only 3 out of 99 cases with POEMS syndrome had heart failure (1). On the other hand, cardiomyopathy is a frequent complication in patients with immunoglobulin light chain (AL) amyloidosis and represents the most definitive prognostic factor (2-4). On electrocardiography, cardiac amyloidosis (CA) shows a low potential in the limb lead and poor $\mathrm{R}$ wave elevation in the chest lead, which is called a pseudo-infarct pattern (3). Echocardiography of patients with CA shows concentric thickening of the ventricle wall and a granular sparkling appearance in the thickened interventricular septum

^ ORCID: Goshi Hagiwara, 0000-0002-2588-9614; Masahisa Arahata, 0000-0002-3759-0724; Kohei Hosokawa, 0000-0002-3423-1523. 
$(2,3)$. Rahman et al. reported that if both low voltage on electrocardiography and an interventricular septal thickness of $>1.98 \mathrm{~cm}$ on echocardiography are present, the diagnosis of CA could be made with $72 \%$ sensitivity and $91 \%$ specificity. In this model, the positive and negative predictive values were $79 \%$ and $88 \%$, respectively (5). Moreover, Liu et al. reported that a higher ratio of septal apical to basal segmental longitudinal peak systolic strain $>2.1$ can differentiate CA from other causes of left ventricular hypertrophy (with $88 \%$ sensitivity, $85 \%$ specificity, $67 \%$ positive predictive value, and $96 \%$ negative predictive value) (6). Accordingly, if these typical physiological findings are evident in a patient with monoclonal gammopathy ( $M$ proteinemia), the patient is usually diagnosed with CA. However, as the management and prognosis of heart failure due to $\mathrm{AL}$ amyloidosis greatly differs from that of heart failure due to other causes, the etiology of cardiomyopathy should be cautiously determined. We experienced a case of POEMS syndrome complicated by congestive heart failure (CHF) that was originally suspected to be CA ineligible for autologous peripheral blood hematopoietic stem cell transplantation (auto-PBSCT); however, it was eventually found that the $\mathrm{CHF}$ was not associated with AL amyloidosis.

We present the following article in accordance with the CARE reporting checklist (available at https://dx.doi. org/10.21037/atm-21-1071).

\section{Case presentation}

A 52-year-old previously healthy man was transferred to our hospital for treatment for suspected POEMS syndrome with a solitary plasmacytoma in the left ischium. Nine months earlier, he noted a difficulty in raising his toes and walking, and stumbling and falling with increased frequency. Four months later, dysarthria was also noted and was admitted to the Department of Neurosurgery of the first hospital to undergo close examinations. Brain magnetic resonance imaging (MRI) showed multiple cerebral infarctions in the occipital and temporal lobes, near the left lateral ventricle. Systemic computed tomography (CT) showed a space-occupying lesion in the left ischium and multiple mild lymphadenopathies in the inguinal, bilateral common iliac arterial, and para-aortic regions, as shown in Figure 1A. No osteosclerotic change was detected by the CT scan. An inguinal lymph node biopsy revealed no specific features suggestive of Castleman disease. IgGlambda-type $\mathrm{M}$ protein was detected; however, plasma cells in the bone marrow accounted for $<10 \%$ of all nucleated cells, which did not meet the diagnostic criteria for multiple myeloma. Three months later, he was transferred to the second hospital to receive a biopsy from the lesion in the ischium. The histopathological findings of the lesion were compatible with plasmacytoma (Figure 1B-1E). A closer examination revealed polyneuritis, hemangiomas at the chest skin, plasmacytoma, IgG-lambda type $M$ protein with elevated serum vascular endothelial growth factor (VEGF, 5 , $150 \mathrm{pg} / \mathrm{mL}$ ), bilateral pleural effusion, and mild hypothyroidism. A physical examination and echocardiography (UCG) revealed remarkable pitting edema on the bilateral lower limbs and a reduced ejection fraction. He had no dyspnea on exertion because he was mostly bedridden due to paralysis of the lower legs. His symptoms were suspected to be caused by POEMS syndrome with a solitary plasmacytoma complicated by CHF; however, neurological symptoms and CHF did not improve after radiotherapy (50 Gy) targeting the plasmacytoma. Moreover, his CHF was refractory to conventional medications such as diuretics, beta-blocker, and angiotensin-converting enzyme inhibitor.

On admission to our hospital, a physical examination showed multiple lymph nodes of $<10$ millimeters in diameter in the bilateral neck and supraclavicular fossa regions, and several small hemangiomas on the chest skin. The muscle strength in both upper limbs was intact, but hypoesthesia was detected in the fingers. The bilateral lower limbs were symmetrically paralyzed on the distal sides. Mild pitting edema was present on the bilateral feet. A nerve conduction study failed to detect any electrical signal from the nerves of the patient's legs. A plain chest X-ray showed a cardiothoracic ratio of $52 \%$ and pleural effusion, predominantly in the left (Figure $2 A$ ). An electrocardiogram (ECG) showed left axis deviation, low voltage in the limb leads and poor $\mathrm{R}$-wave progression in chest leads $\mathrm{V} 1-\mathrm{V} 4$ (Figure 2B). UCG showed a granular sparkling appearance in the thickened interventricular septum (Figure 2C) and an abnormal systolic septal longitudinal base-toapex strain gradient (Figure 2D). Cardiac MRI showed slightly enhanced diffuse ventricular wall at the late phase of gadolinium enhancement (Figure 2E). Based on these findings, we suspected that the patient had CA secondary to $\mathrm{M}$ proteinemia. Further, the amyloidosis was deemed to be extremely advanced and ineligible for auto-PBSCT because the serum $\mathrm{N}$-terminal fragment of pro-B-type natriuretic peptide (NT-proBNP) and troponin T levels were elevated to $12,631 \mathrm{pg} / \mathrm{mL}$ and $0.054 \mathrm{ng} / \mathrm{mL}$, respectively. The 

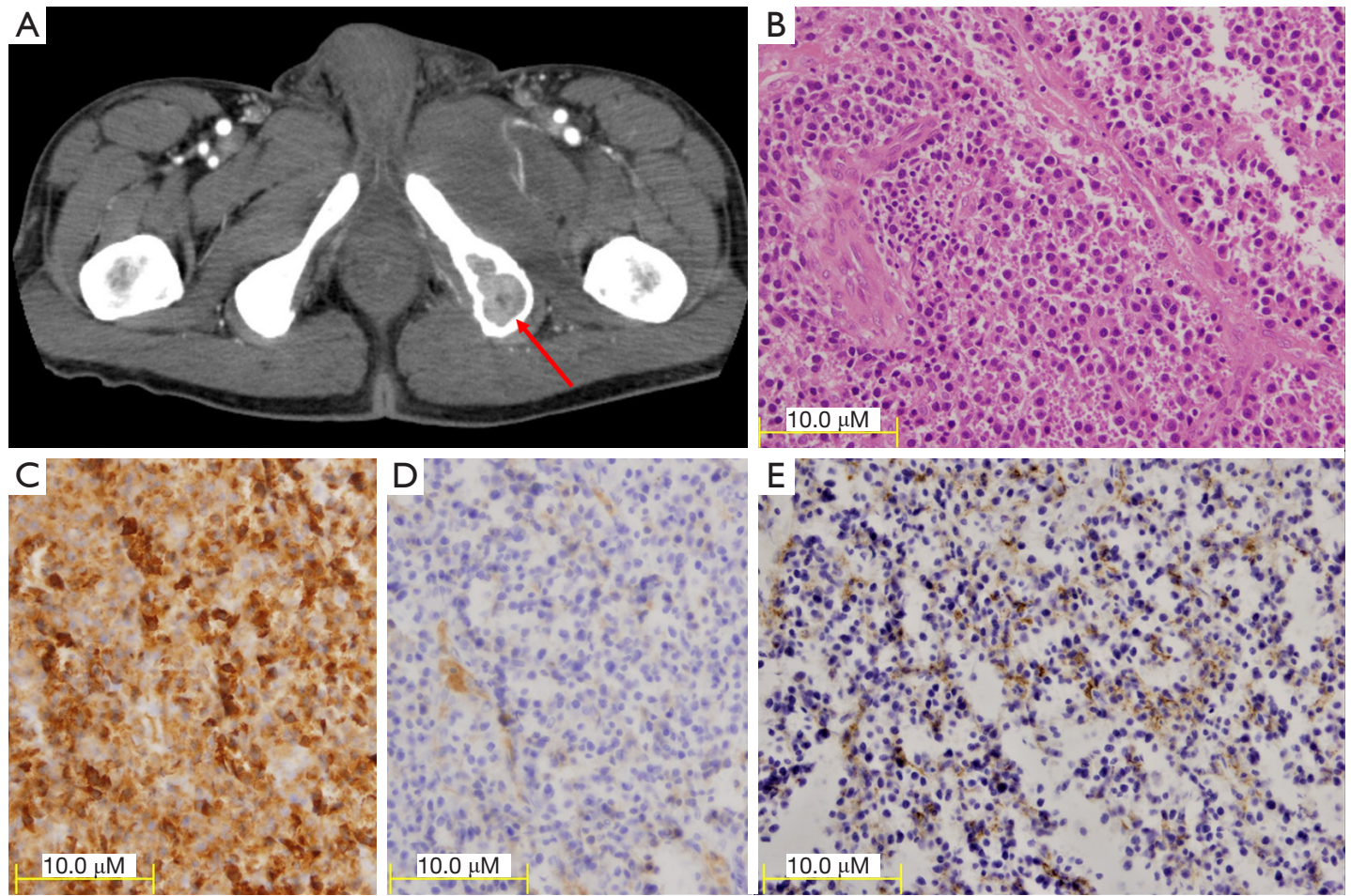

Figure 1 Images of solitary plasmacytoma in the left ischium. (A) The red arrow indicates an occupying lesion in the left ischium on CT. A specimen of this lesion was obtained by needle biopsy. (B-E) Microscopic images of the needle biopsy specimen (×200). Atypical plasma cells were aggregated in the tumor tissue (B; hematoxylin-eosin staining). An immunohistopathological study revealed strong lambda-chain staining (C) but little kappa-chain staining (D). CD138 was also stained in the immunopathological study (E).

concentration of serum free light chain (FLC) lambdatype and FLC ratio were $46.0 \mathrm{mg} / \mathrm{L}$ (reference range; $5.7-26.3 \mathrm{mg} / \mathrm{L}$ ) and 1.43 (reference range; 0.26-1.65), respectively. On the other hand, a myocardial biopsy did not reveal deposits of amyloid proteins either (Figure 3). Additional biopsies from the skin, subcutaneous fat, and iliac bone marrow failed to detect any amyloid deposits. However, the cardiologist in charge of the patient considered the negative result to be a sampling error, based on the patient's ECG, UCG, and MRI findings, which were all typical of CA. Pulmonary hypertension $(\mathrm{PH})$, a frequent complication of POEMS syndrome (7), was not initially suspected because pulmonary valve regurgitation was not detected and the tricuspid regurgitation was too trivial to measure by UCG. On the other hand, right heart catheterization revealed elevated mean pulmonary arterial pressure $(27 \mathrm{mmHg})$, diastolic pulmonary arterial pressure $(18 \mathrm{mmHg})$, and pulmonary capillary wedge pressure $(15 \mathrm{mmHg})$. However, the patient's low pulmonary vascular resistance (1.96 Wood units) and low diastolic pressure gradient $(3 \mathrm{mmHg})$ suggested that his $\mathrm{PH}$ was caused by left heart disease (8).

We selected a chemotherapy regimen with melphalan and dexamethasone (MD) for the treatment of AL amyloidosis because it was less cardiotoxic than other regimens for plasma cell proliferative disorders. Only one cycle of MD therapy dramatically improved the patient's CHF symptoms, as shown in Figure 4. Notably, pitting edema and pleural effusion disappeared rapidly, and NT-proBNP promptly decreased to $346 \mathrm{pg} / \mathrm{mL}$. Given the prompt improvement of the CHF findings, CA was denied as the etiology of his heart failure, and the patient was finally diagnosed with POEMS syndrome, which was eligible for auto-PBSCT after high-dose chemotherapy. After four cycles of MD therapy followed by peripheral blood stem cell harvesting, we switched the induction regimen to lenalidomide and dexamethasone (LD) to intensify the anti-myeloma cell activity. MD and LD were well tolerated. After completing ten cycles of LD, auto-PBSCT combined with high-dose chemotherapy was performed and completed without any serious adverse event. The abnormal findings such as the apical sparing pattern of longitudinal strain on UCG and 

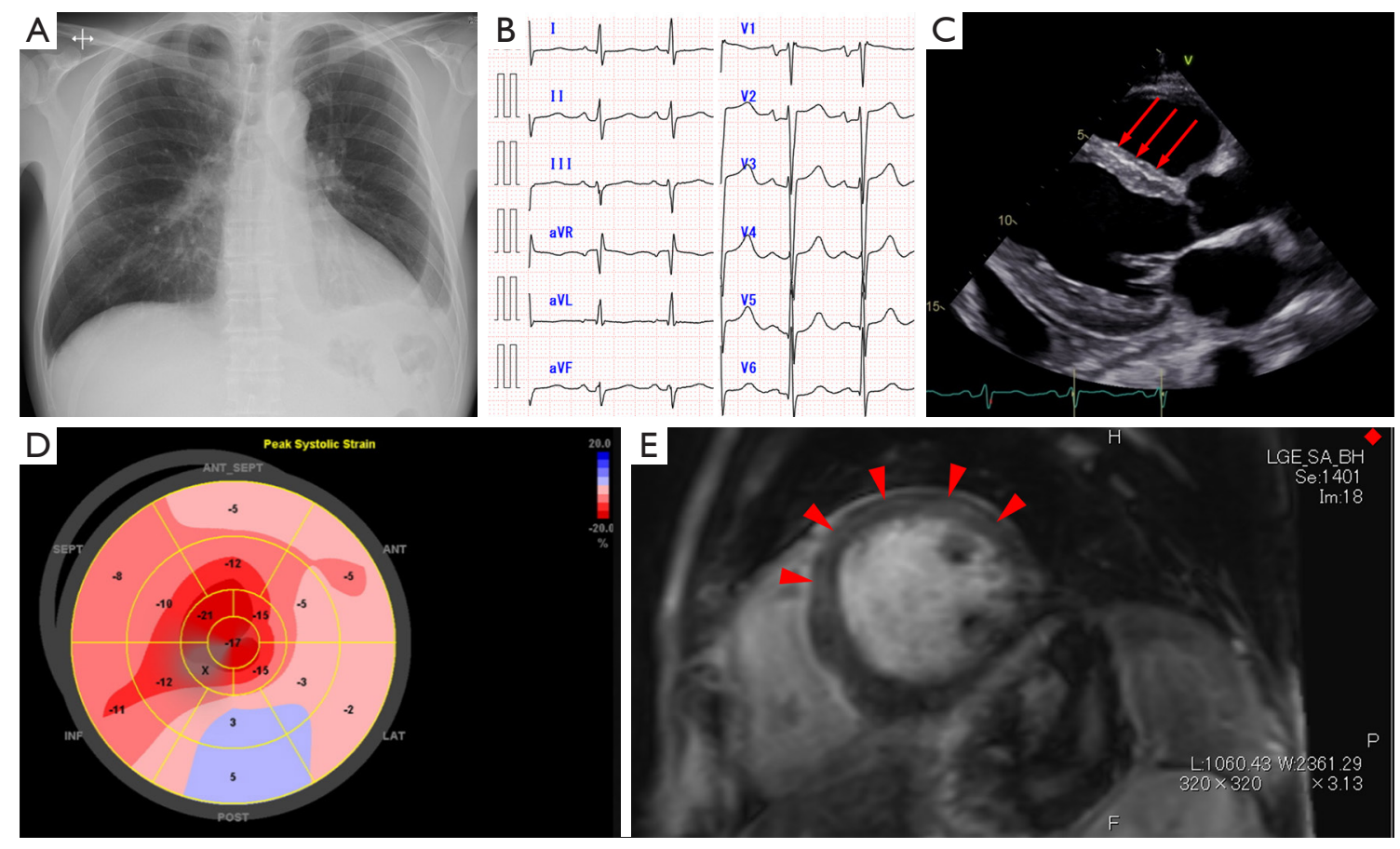

Figure 2 Imaging studies to investigate the cause of heart failure. (A) A plain chest X-ray showed a cardiothoracic ratio of $52 \%$ and pleural effusion predominantly in the left. (B) The electrocardiogram showed left axis deviation, low voltage in the limb leads, and poor R-wave progression in the chest leads V1-V3. (C) Echocardiography showed a reduced ejection fraction (46\%) and granular sparkling appearance seen in the interventricular septum (red arrows), which was $15 \mathrm{~mm}$ in thickness. (D) Speckle tracking echocardiography showed that the septal apical to basal segmental longitudinal peak systolic strain ratio was 4.25 (17/4), indicating an apical sparing pattern. (E) Cardiac magnetic resonance imaging showed late gadolinium enhancement of the left ventricular wall with a weak level (arrow heads).

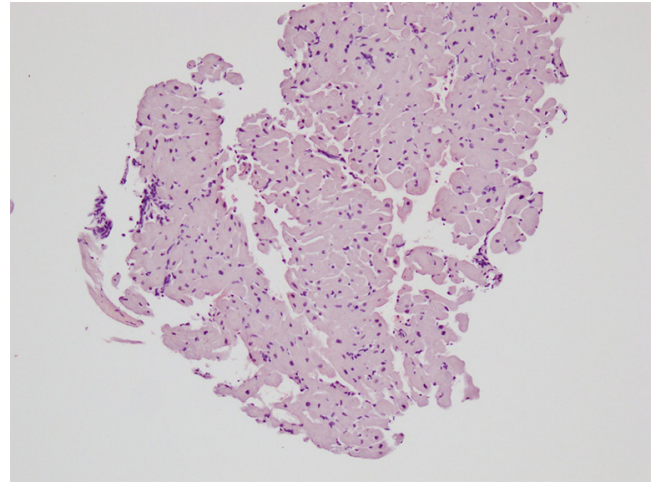

Figure 3 Direct fast scarlet staining of a cardiac biopsy specimen ( $\times 100)$. The biopsy sample was obtained from the right ventricular wall. No amyloid deposit was detected in this specimen.

late gadolinium enhancement of the left ventricular wall on MRI all disappeared. The patient did not develop any signs of cardiac failure and his bilateral lower leg paralysis showed partial resolution. The patient became able to walk on his own after auto-PBSCT, leading to his higher quality of life and deep satisfaction.

All procedures performed in studies involving human participants were in accordance with the ethical standards of the institutional and/or national research committee(s) and with the Helsinki Declaration (as revised in 2013). Written informed consent was obtained from the patient.

\section{Discussion}

In the present case, CHF due to amyloid cardiomyopathy that occurred concurrently with POEMS syndrome was initially suspected because-in addition to $M$ proteinemia-all physiological and laboratory findings, including a low voltage with a pseudo-infarct pattern in the electrocardiogram, the granular sparkling appearance of the thickened cardiac wall on UCG, and the apical sparing pattern of longitudinal strain on speckle tracking UCG were all compatible with those of CA. In addition, this hypothesis was supported by the facts that $\mathrm{CHF}$ is a 


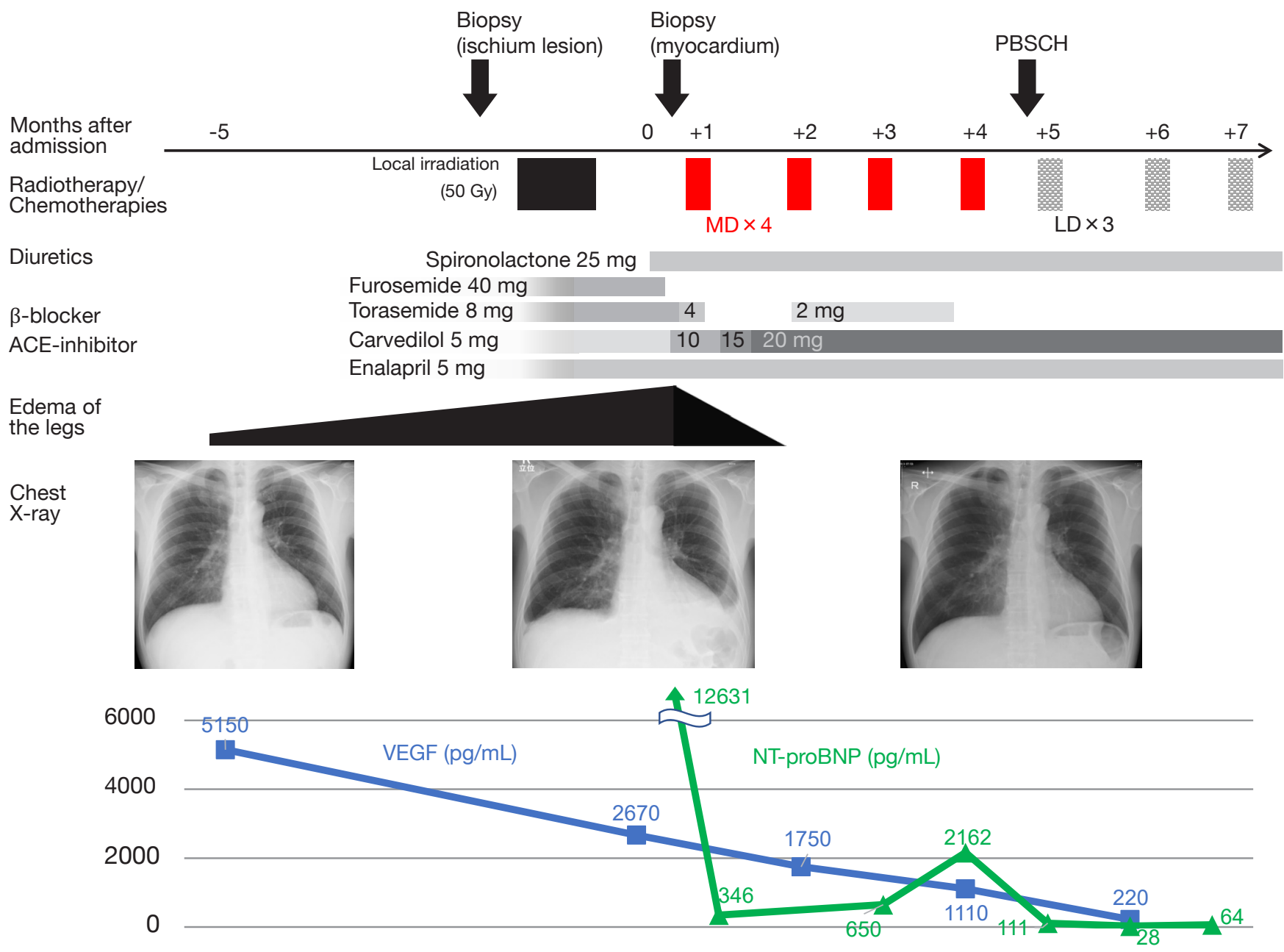

Figure 4 The clinical course of the patient. The patient's CHF rapidly improved during the early phase of MD therapy (oral melphalan $0.22 \mathrm{mg} / \mathrm{Kg} /$ day plus oral dexamethasone $40 \mathrm{mg} /$ day on days 1 to 4 in 28 -day cycles). MD, chemotherapy with melphalan plus dexamethasone; LD, chemotherapy with lenalidomide plus dexamethasone; PBSCH, peripheral blood stem cell harvesting.

rare complication in patients with POEMS syndrome and that CA and POEMS syndrome can coexist $(1,9)$. Although these characteristics have been thought to be specific to CA $(3,5,6)$, the specificity of the diagnostic findings is not $100 \%$. Indeed, the myocardial biopsy of our patient failed to reveal amyloid deposits. However, we did not diagnose any conditions other than CA before chemotherapy because most of the test results were compatible with CAlike features. CA is usually resistant or slowly responsive to chemotherapy regimens such as MD (10) and cannot be corrected by only one cycle of MD therapy. The prompt response of the patient's CHF to MD therapy led us to change the treatment plan for his POEMS syndrome, which eventually helped improve his neurological symptoms.

In recent years, LD has been a preferred treatment for patients with POEMS syndrome who are ineligible for autoPBSCT (11-13). On the other hand, cyclophosphamide, bortezomib, and dexamethasone (CyBorD) or MD are recommended therapies for AL amyloidosis (14). However, CyBorD is reported to be unsuitable for patients with advanced CA with NT-proBNP levels of $>8,500 \mathrm{ng} / \mathrm{L}$ because of its limited efficacy and potential cardiac toxicity $(15,16)$. Thus, prioritizing treatment of CA, we treated the patient with $\mathrm{MD}$, which has minimal cardiac toxicity $(17,18)$. This treatment eventually led to the marked improvement of CHF. Although radiotherapy might have partially 
Table 1 Published case reports of heart failure associated with POEMS syndrome

\begin{tabular}{|c|c|c|c|c|c|c|c|}
\hline Age & Sex & ECG & UCG & CAG & Myocardial biopsy & Treatment & Ref. \\
\hline 31 & M & $\begin{array}{l}\text { Inverted ST segment } \\
\text { and T-wave in } V_{4}-V_{6}\end{array}$ & $\begin{array}{l}\text { Dilatation of left ventricle } \\
\text { hypokinesis }\end{array}$ & $\begin{array}{l}\text { Diffuse } \\
\text { multiple } \\
\text { stenosis }\end{array}$ & $\begin{array}{l}\text { Ischemic } \\
\text { cardiomyopathy }\end{array}$ & $\begin{array}{l}\text { Resection of } \\
\text { plasmacytoma }\end{array}$ & (20) \\
\hline 55 & $\mathrm{~F}$ & NA & $\begin{array}{l}\text { Hypokinesis (Ejection } \\
\text { fraction } 28 \% \text { ) }\end{array}$ & Normal & $\begin{array}{l}\text { Mild hypertrophy } \\
\text { atypical nuclei }\end{array}$ & $\begin{array}{l}\text { Thalidomide plus } \\
\text { dexamethasone } \\
\text { followed by auto- } \\
\text { PBSCT }\end{array}$ & (22) \\
\hline
\end{tabular}

We extracted the cases in which amyloid cardiomyopathy had been excluded by myocardial biopsy. CAG, coronary angiography; ECG, electrocardiogram; MD, melphalan plus dexamethasone therapy; LD, lenalidomide plus dexamethasone therapy; NA, not available; PBSCT, peripheral blood stem cell transplantation; UCG, echocardiography; Ref., reference.

contributed to the improvement of the CHF, this possibility seems unlikely because his CHF progressed during radiotherapy, despite the fact that his serum VEGF level decreased by half. Starting treatment with MD for patients with POEMS syndrome complicated by CHF therefore appears to be useful for excluding CA.

The mechanism underlying $\mathrm{CHF}$ in patients with POEMS syndrome is unclear. An observational study identified only three cases of CHF among 99 enrolled patients with POEMS syndrome (1). All three patients' cardiac symptoms resolved after the treatment of plasma cell dyscrasia with either radiation or combination chemotherapy (1). We searched the PubMed ${ }^{\circledR}$ database for case reports of CHF associated with POEMS syndrome and found only four cases; all four patients underwent a myocardial biopsy that failed to detect amyloid deposition in the myocardial tissues (Table 1) (19-22). Interestingly, the CHF of these patients resolved after the treatment of POEMS syndrome (Table 1). In contrast to our case, CA was not suspected in these patients due to the absence of the typical findings on both ECG and UCG (Table 1). Markedly high serum VEGF levels may predispose patients with POEMS to the development of $\mathrm{CHF}$, which can be reverted by a reduction in the VEGF level after successful treatment of POEMS syndrome.

Although many CA-like features were seen in the patient, some results were consistent with cardiac conditions other than CA. In addition to the normal FLC ratio, the extremely elevated serum NT-proBNP level $(12,631 \mathrm{pg} / \mathrm{mL})$, despite the slightly high troponin $\mathrm{T}$ level $(0.054 \mathrm{ng} / \mathrm{mL})$, may be more indicative of cardiomyopathy associated with POEMS syndrome than of CA. However, no studies on POEMS syndrome have documented such a discrepancy between NT-proBNP and troponin T levels (Table 1) (19-22). Although NT-proBNP and troponin $\mathrm{T}$ have been used to estimate the clinical severity and prognosis of CA (23), these are not diagnostic markers of CA. On the other hand, in their review, Oerlemans et al. suggested that disproportionally high levels of NTproBNP and chronically elevated troponin $\mathrm{T}$ at low levels with normal coronary angiography may be a red flag for CA (24). Additionally, the cardiac MRI findings of our patient may have been equivocal and not highly specific for CA. T1 mapping, which was performed after administration of $\mathrm{MD}$, showed a normal extracellular volume (ECV, $24.1 \%)$. However, the T1/ECV value was not useful for differential diagnosis because the value may have affected by the chemotherapy. To definitely exclude CA, myocardial biopsy is necessary in all cases for whom chemotherapy of plasma cell dyscrasias is planned, even when biopsy results from other organs are compatible with amyloidosis.

The diagnosis of CA in patients with plasma cell 
dyscrasia is challenging because even cardiac biopsies are not $100 \%$ diagnostic due to the difficulty in sampling myocardial tissue (25). If our patient was diagnosed with end-stage CA, the patient may have lost a chance to undergo auto-PBSCT, which is the most effective treatment for POEMS syndrome (10). It is therefore important for physicians not to assume that $\mathrm{CHF}$ in patients with plasma cell dyscrasia is associated with AL amyloidosis, even if their laboratory findings are typical for AL amyloidosis, and to perform closer examinations, including myocardial biopsy.

\section{Acknowledgments}

We would like to thank Mr. Hiroyuki Hayashi, a radiological technician of the Radiology Division in Kanazawa University Hospital, for analyzing cardiac MRI and making a report of T1 mapping of the patient.

Funding: None.

\section{Footnote}

Reporting Checklist: The authors have completed the CARE reporting checklist. Available at https://dx.doi. org/10.21037/atm-21-1071

Peer Review File: Available at https://dx.doi.org/10.21037/ atm-21-1071

Conflicts of Interest: All authors have completed the ICMJE uniform disclosure form (available at https://dx.doi. org/10.21037/atm-21-1071). The authors have no conflicts of interest to declare.

Ethical Statement: The authors are accountable for all aspects of the work in ensuring that questions related to the accuracy or integrity of any part of the work are appropriately investigated and resolved. All procedures performed in studies involving human participants were in accordance with the ethical standards of the institutional and/or national research committee(s) and with the Helsinki Declaration (as revised in 2013). Written informed consent was obtained from the patient.

Open Access Statement: This is an Open Access article distributed in accordance with the Creative Commons Attribution-NonCommercial-NoDerivs 4.0 International License (CC BY-NC-ND 4.0), which permits the noncommercial replication and distribution of the article with the strict proviso that no changes or edits are made and the original work is properly cited (including links to both the formal publication through the relevant DOI and the license). See: https://creativecommons.org/licenses/by-nc-nd/4.0/.

\section{References}

1. Dispenzieri A, Kyle RA, Lacy MQ, et al. POEMS syndrome: definitions and long-term outcome. Blood 2003;101:2496-506.

2. Gertz MA, Dispenzieri A. Systemic Amyloidosis Recognition, Prognosis, and Therapy: A Systematic Review. JAMA 2020;324:79-89.

3. Kitaoka H, Izumi C, Izumiya Y, et al. JCS 2020 Guideline on Diagnosis and Treatment of Cardiac Amyloidosis. Circ J 2020;84:1610-71.

4. Merlini G, Palladini G. Light chain amyloidosis: the heart of the problem. Haematologica 2013;98:1492-5.

5. Rahman JE, Helou EF, Gelzer-Bell R, et al. Noninvasive diagnosis of biopsy-proven cardiac amyloidosis. J Am Coll Cardiol 2004;43:410-5.

6. Liu D, Hu K, Niemann M, et al. Effect of combined systolic and diastolic functional parameter assessment for differentiation of cardiac amyloidosis from other causes of concentric left ventricular hypertrophy. Circ Cardiovasc Imaging 2013;6:1066-72.

7. Li J, Tian Z, Zheng HY, et al. Pulmonary hypertension in POEMS syndrome. Haematologica 2013;98:393-8.

8. Galiè N, Humbert M, Vachiery JL, et al. 2015 ESC/ERS Guidelines for the diagnosis and treatment of pulmonary hypertension: The Joint Task Force for the Diagnosis and Treatment of Pulmonary Hypertension of the European Society of Cardiology (ESC) and the European Respiratory Society (ERS): Endorsed by: Association for European Paediatric and Congenital Cardiology (AEPC), International Society for Heart and Lung Transplantation (ISHLT). Eur Heart J 2016;37:67-119.

9. Adami F, Briani C, Binotto G, et al. Coexistence of primary AL amyloidosis and POEMS syndrome: efficacy of melphalan-dexamethasone and role of biochemical markers in monitoring the diseases course. Am J Hematol 2010;85:131-2.

10. Manwani R, Foard D, Mahmood S, et al. Rapid hematologic responses improve outcomes in patients with very advanced (stage IIIb) cardiac immunoglobulin light chain amyloidosis. Haematologica 2018;103:e165-8.

11. Dispenzieri A. POEMS Syndrome: 2019 Update on diagnosis, risk-stratification, and management. Am J 
Hematol 2019;94:812-27.

12. Nozza A, Terenghi F, Gallia F, et al. Lenalidomide and dexamethasone in patients with POEMS syndrome: results of a prospective, open-label trial. Br J Haematol 2017;179:748-55.

13. Zhao H, Huang XF, Gao XM, et al. What is the best first-line treatment for POEMS syndrome: autologous transplantation, melphalan and dexamethasone, or lenalidomide and dexamethasone? Leukemia 2019;33:1023-9.

14. National Comprehensive Cancer Network [Internet]. NCCN Clinical Practice Guidelines in Oncology (NCCN Guidelines $\left.{ }^{\circledR}\right)$ : Systemic Light Chain Amyloidosis, Version 2.2021-February 8, 2021 [cited 2021 June 1]. Available online: https://www.nccn.org/professionals/physician_gls/ pdf/amyloidosis.pdf

15. Palladini G, Sachchithanantham S, Milani P, et al. A European collaborative study of cyclophosphamide, bortezomib, and dexamethasone in upfront treatment of systemic AL amyloidosis. Blood 2015;126:612-5.

16. Cole DC, Frishman WH. Cardiovascular Complications of Proteasome Inhibitors Used in Multiple Myeloma. Cardiol Rev 2018;26:122-9.

17. Palladini G, Milani P, Foli A, et al. Oral melphalan and dexamethasone grants extended survival with minimal toxicity in AL amyloidosis: long-term results of a risk-

Cite this article as: Hagiwara G, Arahata M, Hosokawa K, Shimojima M, Nakao S. Congestive heart failure associated with POEMS syndrome that was adequately distinguished from cardiac amyloidosis: a case report and literature review. Ann Transl Med 2021;9(15):1266. doi: 10.21037/atm-21-1071 adapted approach. Haematologica 2014;99:743-50.

18. Jaccard A, Moreau P, Leblond V, et al. High-dose melphalan versus melphalan plus dexamethasone for AL amyloidosis. N Engl J Med 2007;357:1083-93.

19. Shimizu N, Goya M, Akimoto H, et al. Cardiomyopathy in a case of Crow-Fukase syndrome. Jpn Heart J 1997;38:877-80.

20. Ichikawa Y, Nakata T, Ohhata J, et al. Crow-Fukase syndrome with ischemic cardiomyopathy. Intern Med 2001;40:726-30.

21. Iwasaki H, Ogawa K, Yoshida H, et al. Crow-Fukase syndrome associated with pulmonary hypertension. Intern Med 1993;32:556-60.

22. Inoue D, Kato A, Tabata S, et al. Successful treatment of POEMS syndrome complicated by severe congestive heart failure with thalidomide. Intern Med 2010;49:461-6.

23. Pregenzer-Wenzler A, Abraham J, Barrell K, et al. Utility of Biomarkers in Cardiac Amyloidosis. JACC Heart Fail 2020;8:701-11.

24. Oerlemans MIFJ, Rutten KHG, Minnema MC, et al. Cardiac amyloidosis: the need for early diagnosis. Neth Heart J 2019;27:525-36.

25. van Gameren II, Hazenberg BP, Bijzet J, et al. Diagnostic accuracy of subcutaneous abdominal fat tissue aspiration for detecting systemic amyloidosis and its utility in clinical practice. Arthritis Rheum 2006;54:2015-21. 